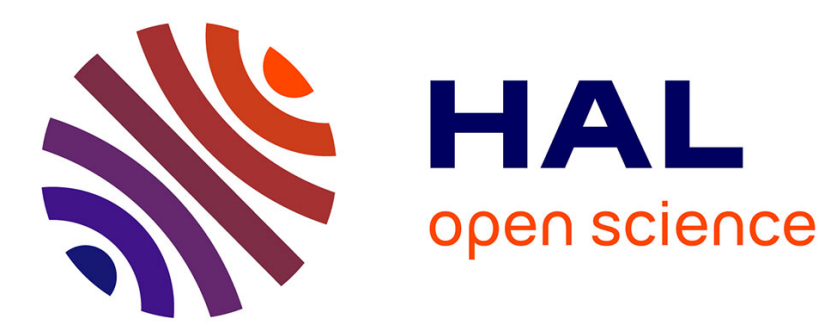

\title{
Cancer cell migration on 2D deformable substrates
}

\author{
Valentina Peschetola, Claude Verdier, Alain Duperray, Davide Ambrosi
}

\section{To cite this version:}

Valentina Peschetola, Claude Verdier, Alain Duperray, Davide Ambrosi. Cancer cell migration on 2D deformable substrates. L. Preziosi and A. Chauvière and C. Verdier. Cell Mechanics: from single scale-based models to multiscale modeling, Chapman \& Hall/CRC, pp.1-24, 2009. hal-00410151

\section{HAL Id: hal-00410151 \\ https://hal.science/hal-00410151}

Submitted on 18 Aug 2009

HAL is a multi-disciplinary open access archive for the deposit and dissemination of scientific research documents, whether they are published or not. The documents may come from teaching and research institutions in France or abroad, or from public or private research centers.
L'archive ouverte pluridisciplinaire HAL, est destinée au dépôt et à la diffusion de documents scientifiques de niveau recherche, publiés ou non, émanant des établissements d'enseignement et de recherche français ou étrangers, des laboratoires publics ou privés. 


\section{Chapter 9}

\section{Cancer cell migration on 2D}

\section{deformable substrates}

Valentina Peschetola, Claude Verdier, Alain Duperray and Davide Ambrosi

\subsection{Introduction}

Cell migration is an important feature occurring during many biological processes such as tissue invasion, the immunologic response, etc. and involves sophisticated mechanisms such as the development of focal adhesions, possible extra-cellular matrix (ECM) degradation, and activation of the actin-myosin complex, in order to develop forces necessary for traction. Depending on their type and the environment, cells can move according to different types of locomotion, either in 2D or 3D. For example fibroblasts move on a 2D substrate in the most conventional way, as described by Sheetz [34]: they form a lamellipodium at the front, and develop adhesion complexes so that they can pull on such anchors in order to detach the rear part and move forward. On the other hand, fish keratocytes migrating on 2D substrates take the form of a crescent [6, 22]. In three dimensions, two types of motions have been observed for cancer cells migrating in 3D collagen, the "amoeboid" type and the 
"mesenchymal" one $[15,16]$.

2D-migration can depend on cell type and ligand matrix density, as shown in previous works on substrates covered by different densities of ECM [27]. The migration velocity vs. ligand density curve usually exhibits a bell-shaped curve with a maximum velocity at an intermediate ligand density, which is the signature of an optimum affinity between receptor and ligand molecules. This is the basis for understanding the complex machinery provided by cells developing focal adhesions at the front while removing them at the back in the conventional five-step motion [34]. To better understand these features, fluorescence (confocal) microscopy is a very useful tool to show the precise location of adhesion complexes involved during cell migration. The formation of such focal contacts [4] allows the development of important forces that the cell uses to move forward. Such forces are usually called "traction forces" and are difficult to determine by straightforward analysis. Therefore, many attempts have been made in the past ten years to measure such forces in an indirect way and different techniques have been developed in this respect:

- the original technique of Harris and co-workers [18] consisting of observing the magnitude and shape of wrinkles on a soft elastic film below a cell;

- the most classical one by Dembo and co-authors $[9,10]$ measures the motion of fluorescent beads embedded in a gel deformed by a cell;

- micro-patterned substrates $[4,12,35]$ with thin poles whose deflection directly gives the forces exerted by the cell.

All these techniques have been tested in vitro and were quite effective for the study of the motion of cells on 2D-rigid substrates. The first technique [18] was initially rather qualitative, but further developments by Burton et al. [6], in particular devoted to the study of keratocytes locomotion, revealed to be quite quantitative. The last one, introduced by Balaban et al. [4], has received recently a lot of interest [17] and important results concerning cell migration of $3 \mathrm{~T} 3$ fibroblasts on rigid substrates have been obtained regarding the influence of substrate rigidity. Indeed, as earlier understood by Lo et al. [24], cells usually exert larger traction forces on more rigid substrates, this behavior being correlated with an 
increased contact surface and a reduced migration velocity. The traction force increases for larger stiffnesses until a critical rigidity is reached (around $80 \mathrm{kPa}$ ), where the force exhibits a plateau [17]. Although very elegant, the technique due to Balaban et al. still poses questions like whether cells behave similarly on micro-patterned substrates as compared to classical polyacrylamide gels or when migrating in vivo.

In this chapter, we focus on the technique developed by Dembo and Wang [10], and its further recent developments $[2,3,25,30]$. The basic idea is to measure the displacements of fluorescent beads embedded in rigid substrates of polyacrylamide (a mixture of acrylamide and bis-acrylamide to be more precise). Component concentrations can be tuned to obtain the desired gel rigidity, usually ranging between $1 k P a$ and $100 k P a$. As cells are laid onto the functionalized substrate, they adhere and start to exert tractions which deform the substrate so that fluorescent beads located in the underlying gel are displaced. Based on the hypothesis that the gel is isotropic and elastic, and displacements are small enough so that linear elasticity applies, the gel depth being large enough so that it can be approximated by a half space, the Boussinesq equations can be solved in the whole gel. Usually a thickness of $100 \mu \mathrm{m}$ is enough, but a recent work argues about the advantages of using thinner gels [25], so that one can compute the traction stresses exactly at the gel surface. This is an example of inverse problem, since the particle displacement vectors $\mathbf{u}(\mathbf{x}, t)$ are known at certain locations (with an unavoidable experimental error) and the traction stresses $\mathbf{T}(\mathbf{x}, t)$ are to be computed everywhere on the gel surface. The solution of the Boussinesq problem for an elastic gel with Young modulus $E$ and Poisson coefficient $\nu$ reads:

$$
\mathbf{u}(\mathbf{r})=\int \mathbf{G}\left(\mathbf{r}-\mathbf{r}^{\prime}\right) \mathbf{T}\left(\mathbf{r}^{\prime}\right) d \mathbf{r}^{\prime}
$$

where the kernel $\mathbf{G}$ is a Green's function tensor [21]:

$$
\mathbf{G}(\mathbf{r})=\frac{1+\nu}{\pi E}\left((1-\nu) \frac{\mathbf{1}}{r}+\nu \frac{\mathbf{r} \otimes \mathbf{r}}{r^{3}}\right)
$$

while $r$ denotes the modulus of $\mathbf{r}$.

In the initial approach of Dembo and Wang [10], beads displacements are first deter- 
mined. Bi-linear shape functions are defined on an unstructured quadrilateral mesh. The optimal stress field is obtained minimizing the error by an iterative procedure under force magnitude penalization. Since the method is rather complicated than other methods have been introduced [7] making use of the particular structure of the equations. Noticing that Eq.(9.1) is a convolution, one can compute the Fourier transform $\tilde{\mathbf{F}}(\mathbf{k})=\int_{-\infty}^{\infty} e^{-i \mathbf{k} . \mathbf{r}} \mathbf{F}(\mathbf{r}) d \mathbf{r}$ on both sides of Eq.(9.1), $\mathbf{k}$ being the wave vector:

$$
\tilde{\mathbf{u}}(\mathbf{k})=\tilde{\mathbf{G}}(\mathbf{k}) \tilde{\mathbf{T}}(\mathbf{k})
$$

Following the approach of [7], the expression of $\tilde{\mathbf{G}}(\mathbf{k})$ is given by a two-by-two matrix, assuming that vertical displacements can be neglected. This is usually the case as long as gels are stiff enough and are not deformed by vertical tractions exerted by cells, which is a good enough hypothesis in this case. Finally Eq.(9.3) can be inverted to obtain $\tilde{\mathbf{T}}(\mathbf{k})$ and then going back into the real space gives the traction stress field $\mathbf{T}(\mathbf{r})$ :

$$
\mathbf{T}(\mathbf{r})=F T_{2}^{-1}\left(\tilde{\mathbf{G}}^{-1}(\mathbf{k}) \tilde{\mathbf{u}}(\mathbf{k})\right)
$$

where $F T_{2}^{-1}$ denotes the two-dimensional inverse Fourier Transform and $\tilde{\mathbf{G}}^{-\mathbf{1}}(\mathbf{k})$ is the inverse of $\tilde{\mathbf{G}}(\mathbf{k})$ which can be computed explicitly thanks to simple manipulations [7]. The main drawback of this method is that despite its simplicity, it requires to have a periodic displacement field in order to compute the Fast Fourier Transforms.

One further work [33] deals with pointwise traction force reconstruction and the effect of noise, which are well known to affect the results. Due to the ill-posedness of the problem, it is necessary to use a regularization algorithm to obtain significant data. Another information that can be used is the location of focal adhesions. Another possible hypothesis [25] is to use thin substrates (typically $10 \mu \mathrm{m}$ in thick) which can help get explicit solutions of the Boussinesq equations. This allows an increased accuracy in the determination of these traction stresses. To conclude, let us mention the recent work of Sabass et al. [30] who combined three approaches to determine traction forces, one based on the integral boundary element method (BEM) [10], one using the Fourier Transform Traction Cytometry (FTTC) 
[7], and the last one mentioned just above using Traction Recovery with Point Forces (TRPF) [33]. All methods can be improved when proper regularizations are used and can lead to an increased resolution. The TRPF procedure seems to be the most promising one, as long as focal adhesions are well developed, but requires of course more sophisticated experiments. These experiments are needed in any case, as one wants to clearly understand how forces are connected with focal adhesions, and also how the cell cytoskeleton plays a role in the different motility regimes.

In this chapter, a different method $[2,3]$ is used as a promising tool for studying the migration of cancer cells. It is based on a formulation arising from the minimization of an energy, combined with the use of a penalty parameter. The method will be presented in the next section. Then detailed experimental methods will be given. The results showing displacements and stresses in the case of migration T24 cancer cells will be eventually presented. The discussions will concern the effect of gel rigidity, as well as comparisons with other cell types.

\subsection{An adjoint method for cell traction}

An alternative approach to obtain the pattern of the shear stresses exerted by a cell on a flat substrate is the adjoint method proposed by Ambrosi $[2,3]$. The mathematical model is based on the classical functional analysis framework due to Lions [23]; the general theory is applied to the specific problem of small deformation of a homogeneous elastic material subjected to body forces only.

Let $\Omega$ be the whole domain and $\mathbf{u}(\mathbf{x})$ the displacement vector field, $\mathbf{x} \in \Omega \subset \mathbb{R}^{3}$. The displacement is known only in a subset $\Omega_{0} \subset \Omega$ where beads are located; the related function $\mathbf{u}_{0}(\mathbf{x})$ has support in $\Omega_{0}$. Let $\Omega_{c} \subset \Omega$ be the region covered by the cell and where the shear stress is applied. As explained before, the traction forces are generated through the actinmyosin interactions and act on the underlying substrate through focal adhesion sites. These areas are not localized precisely in our experiments and we do not restrict the force support to these sites as is done in the algorithm of Schwarz et al. [33]. 
Consider the following elastic problem in the whole domain $\Omega$

$$
-\mu \Delta \mathbf{u}-(\mu+\lambda) \nabla(\nabla \cdot \mathbf{u})=\mathbf{f},\left.\quad \mathbf{u}\right|_{\partial \Omega}=0
$$

where $\mu$ and $\lambda$ are the Lamé constants that characterize the material. The problem can be rewritten in the form $A \mathbf{u}=\mathbf{f}$, where $\mathrm{A}$ is a linear operator which is deduced easily from the above equation. The aim is to obtain the force field $\mathbf{f}$, that is inferred by a known displacement (inverse problem). If we try to invert directly the equation, we find that the problem is ill-posed since the displacement is known only in a subset $\Omega_{0}$ of $\Omega$. It is necessary to introduce the projector $P, P: \Omega \rightarrow \Omega_{0}$, and a functional $J(\mathbf{f}), J: L^{2}(\Omega) \rightarrow \mathbb{R}$, defined as

$$
J(\mathbf{f})=\int_{\Omega_{0}}\left|\mathbf{u}-\mathbf{u}_{0}\right|^{2} d V+\varepsilon \int_{\Omega}|\mathbf{f}|^{2} d V
$$

where $\varepsilon$ is a real positive number. This functional measures the difference between the displacement field produced by $\mathbf{f}$ and the experimental one defined by $\mathbf{u}_{0}$ under penalization of the square norm of the force field itself. We look for $\mathbf{g}$ minimizing $J$ :

$$
J(\mathbf{g}) \leq J(\mathbf{f}), \quad \forall \mathbf{f} \in V_{c}
$$

where $V_{c} \subset L^{2}(\Omega)$ is the space of the finite energy functions with support in $\Omega_{c}$. The minimization of $J$ accomplishes the minimization of the distance of the solution from the measured value $\mathbf{u}_{0}$ under penalization of the magnitude of the associated force $\mathbf{f}$ per unit surface. The penalty parameter $\varepsilon$ balances the two requirements. An equivalent condition of (9.7) is given by $J^{\prime}(\mathbf{g})[\mathbf{f}-\mathbf{g}] \geq 0$; making the Gateaux derivative explicit suggests the introduction of the adjoint equations, $A^{*}: L^{2}(\Omega) \rightarrow \Omega_{0}$

$$
A^{*} \mathbf{q}=P \mathbf{u}(\mathbf{g})-\mathbf{u}_{0},\left.\quad \mathbf{q}\right|_{\partial \Omega}=0
$$

Substituting back in the functional derivative permits to obtain the solution of (9.7) that represents the optimal body force:

$$
\mathbf{g}=-\frac{\chi_{c}}{\varepsilon} \mathbf{q}
$$


$\chi_{c}$ is the characteristic function of the domain $\Omega_{c}$ and $\mathbf{q}$ is a volume force.

In short, the set of equations that we need to solve is given by two elliptic partial differential equations, one for the displacement $\mathbf{u}$ and the adjoint one for the volume force q.

On the basis of dimensional arguments, it is possible to reduce this three-dimensional system (in space) into a two-dimensional one. In fact, vertical averaging along an effective thickness $h$ allows to introduce two parameters $\hat{\mu}$ and $\hat{\lambda}$ :

$$
\hat{\mu}=h \frac{E}{2(1+\nu)}, \quad \hat{\lambda}=h \frac{E \nu}{1-\nu^{2}} .
$$

$E$ and $\nu$ are the Young modulus and the Poisson ratio respectively. $h$ is the averaging height fixed by the depth of field of the microscope that is 1.5 microns in our case. Below this depth, the beads are not in focus and their positions are not measured; the displacement $\mathbf{u}$ should be understood as the average displacement along $h$, which is nearly the displacement of the center of the beads. Finally, the two-dimensional system becomes

$$
\begin{aligned}
& -\hat{\mu} \Delta \mathbf{u}-(\hat{\mu}+\hat{\lambda}) \nabla(\nabla \cdot \mathbf{u})=-\frac{\chi_{c}}{\varepsilon} \mathbf{p},\left.\quad \mathbf{u}\right|_{\partial \Omega}=0, \\
& -\hat{\mu} \Delta \mathbf{p}-(\hat{\mu}+\hat{\lambda}) \nabla(\nabla \cdot \mathbf{p})=\chi_{o} \mathbf{u}-\mathbf{u}_{0},\left.\quad \mathbf{p}\right|_{\partial \Omega}=0,
\end{aligned}
$$

where $\chi_{c}$ and $\chi_{0}$ are the characteristic functions related to $\Omega_{c}$ and $\Omega_{0}$ respectively. Note that the structure of the two equations is the same because the problem is self-adjoint. $\mathbf{p}$ now represents the traction stresses $\left(N / m^{2}\right)$ previously introduced as $\mathbf{T}$ and will be determined once the two equations are solved.

In the ill-posed problem the penalty parameter, $\varepsilon$ in our case, plays an important role. In order to fix $\varepsilon$ it is necessary to re-interpret the system (9.9) on the basis of arguments suggested by modal analysis. Suppose that $\Omega_{0}=\Omega_{c}=\Omega$ under periodic boundary conditions, the previous system of equations rewrites like a Tikhonov filter. The amplitude of the 
Fourier components of the solution $u_{k}, p_{k}$ satisfy the algebraic relations

$$
\begin{aligned}
h E k^{2} u_{k} & \simeq-\frac{1}{\varepsilon} p_{k}, \\
h E k^{2} p_{k} & \simeq u_{k}-u_{0, k},
\end{aligned}
$$

that is

$$
u_{k} \simeq \frac{u_{0, k}}{1+\varepsilon h^{2} E^{2} k^{4}}
$$

where $u_{0, k}$ represents the amplitude of the $k$-th Fourier component of $u_{0}$. According to equation (9.11), if the data is known all over the domain the system of equations (9.9) is a filter damping the modes corresponding to wavenumbers $k>\varepsilon^{-1 / 4} h^{-1 / 2} E^{-1 / 2}$. The choice of $\varepsilon$ can be interpreted in terms of filtering modes falling below the experimental accuracy. Equation (9.11) shows that the key parameter of the inversion procedure is actually $\varepsilon h^{2}$ and the solution does not change for combinations of the averaging layer $h$ and penalty parameter $\varepsilon$ that preserve this quantity.

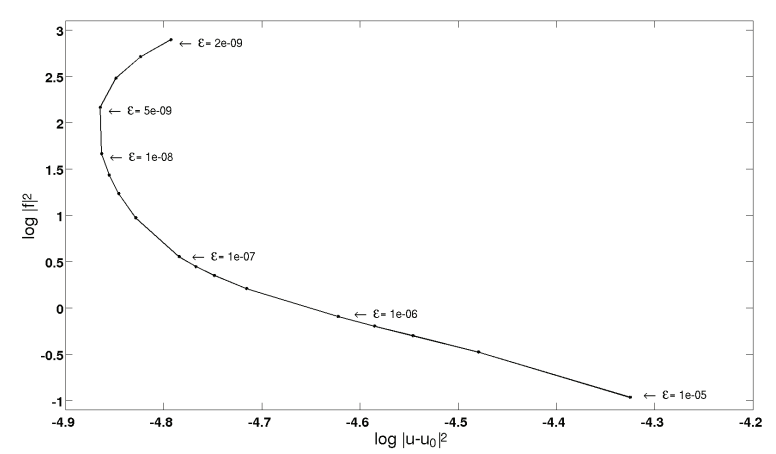

Figure 9.1: Discrete L-curve with 17 values of $\varepsilon$ obtained for our model in the case of the stiff gel $(E=10 k P a)$. The corner corresponds to the optimal balance between data agreement and regularization.

A convenient tool for the analysis of discrete ill-posed problems is the L-curve criterion which is a log-log plot, for all valid regularization parameters, of the norm of the regularized solution $\|\mathbf{p}\|_{2}$ versus the corresponding residual norm $\left\|\mathbf{u}-\mathbf{u}_{0}\right\|_{2}$. In this way, the L-curve displays the minimization of these two quantities and its corner, which is intrinsic to the data, corresponds to the optimal balance between data agreement and regularization. Figure 9.1 shows an example of the discrete L-curve obtained for 17 values of the $\varepsilon$ parameter for our model in the case of the stiff gel $(E=10 k P a)$. In our work we take the minimum value 
of $\varepsilon$ that does not yield erratic results in the displacement, that is the corner of the L-curve.

The system of equations (9.9) has been discretized by a finite element method using linear basis functions on an unstructured mesh. In order to avoid any iterative coupling between the two equations in (9.9), a global conjugate method has been used to solve the resulting system of linear equations numerically. Figure 9.2 shows an example of the computational setting: the computational mesh, the beads displacement and the cell contour. The triangular mesh satisfies two constraints: it has a node in every point where displacements are known (beads location) and a sequence of element sides coincides with the cell contour. In particular, the boundary $\Omega_{c}$ of the cell, is described as a piecewise linear curve following the shape of the cell and represents the boundary between the between the intracellular domain over the substrate and the rest of the domain where the forces are not applied.

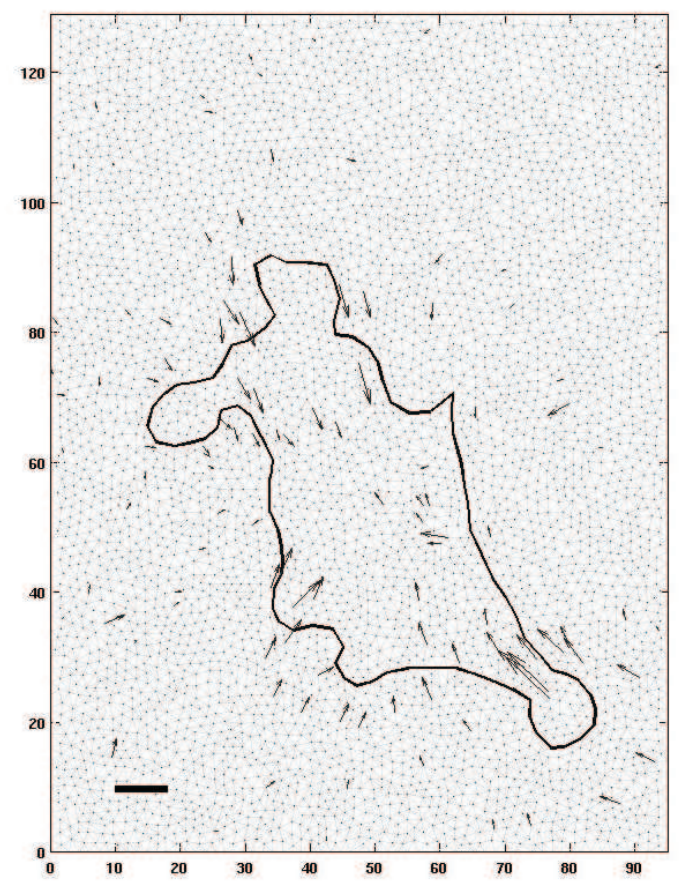

Figure 9.2: Graphical representation of the numerical set-up: the computational mesh, made of triangles, is represented in light grey. The mesh satisfies two constraints: it has one node at every point where the displacement is known and a sequence of element sides coincides with the boundary of the cell. The arrows indicate the experimental beads displacement. The scale bar at the bottom left corner is 0.5 micron long. 


\subsection{Experimental methods}

In this work, we characterize cell migration on gels with different rigidities. T24 epithelial cells from a bladder cancer line are used. This type of cancer cells is known to be rather invasive. Regarding gel preparation, the following experimental procedure is used:

- Gel preparation and stiffness. In order to prepare gels with different stiffnesses, we change the ratio between polyacrylamide and bis-acrylamide components. Three different gels have been prepared containing $5-7.5-10 \%$ of polyacrylamide and the bis-acrylamide percentage is $0.03 \%$. The mechanical properties have been measured using conventional dynamic shear rheometry tests on a Malvern rheometer (Gemini 150, stress-controlled). Sinusoidal oscillations at a known shear deformation $\gamma=\gamma_{0} \sin (\omega t)$ are applied within the linear regime (small enough deformation $\gamma_{0} \sim 0.01$ ) at different angular frequencies $\omega$. The stress response $\sigma=\sigma_{0} \sin (\omega t+\phi)$ (where $\sigma_{0}$ is a constant stress and $\phi$ is the phase angle) is measured and the elastic $\left(G^{\prime}\right)$ and viscous moduli $\left(G^{\prime \prime}\right)$ are deduced. These gels are quite elastic, as long as the acrylamide concentration is large enough, which is the case. Indeed, experiments show a constant elastic modulus $G^{\prime}$ when the frequency $f$ (related to $\omega$ by $\omega=2 \pi f$ ) ranges from 0.1 to $10 \mathrm{~Hz}$. The loss modulus $G^{\prime \prime}$ is usually lower by two orders of magnitude. Therefore we can assume that $\nu=0.5$, for an incompressible gel, thus $E=3 G^{\prime}$. Note that the hypothesis that $E=3 G^{\prime}$ is relevant here in comparison to the work of Boudou et al. [5] showing that $\nu \sim 0.48$ in such polyacrylamide gels, as determined using micropipettes. This leads to the typical gel Young's moduli $2 k P a, 6.3 k P a$ and $10 k P a$ for respectively the soft, medium and hard gels which are used in this study. An example of the influence of the polyacrylamide content (for the three gels) is shown in Fig.9.3. The typical slope of $\sim 2$ is current in such biological networks as observed for example in actin and fibrin gels [20]. Comparisons have been made previously [3] with other authors [5, 13, 14, 37] and revealed good qualitative agreement.

- Methodology. Gels were prepared on a silanated square coverglass $22 \mathrm{~mm}$ x $22 \mathrm{~mm}$, covered with a circular coverglass (35 $\mathrm{mm}$ diameter) and treated with $\mathrm{NaOH}(0.1 \mathrm{M})$, (3-Aminopropyl)trimethoxysilane (APTMS, $10 \mathrm{mn}$ ), and $0.5 \%$ glutaraldehyde $(30 \mathrm{mn})$. 


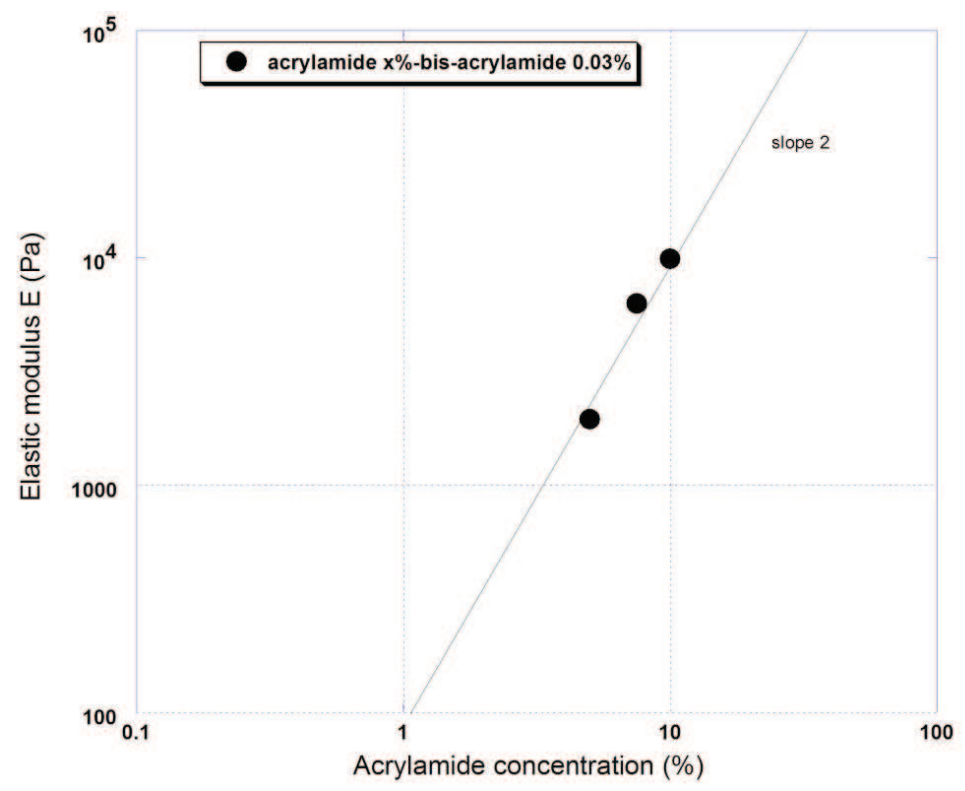

Figure 9.3: Elastic moduli $\mathrm{E}(\mathrm{Pa})$ as a function of the acrylamide concentration. Bisacrylamide concentration is fixed at $0.03 \%$.

Fluorescent beads (Molecular Probes, 0.2 micrometer in diameter) were seeded before addition of the cross-linker. Gels were laid onto the square coverglass, and the circular coverglass was brought carefully to capture the gel by capillarity, this avoiding to flip the preparation. Indeed beads need to sediment fast so that there are located closer to the gel upper surface where measurements are made. The gel volume was chosen so that gels have a thickness of about $70 \mu \mathrm{m}$, as described in other works [28]. The gel was left to polymerize for nearly 90 minutes.

- Gel functionalization. After polymerization, the square coverglass was gently removed and sulfo-Sanpah 1mM mixed with DiMethylSulfOxide (DMSO) and Phosphate Buffered Saline(PBS) was added to functionalize the gel (15 minutes under UV). This procedure was repeated twice, then the surface was rinsed with PBS. Finally a $20 \mu \mathrm{g} / \mathrm{ml}$ fibronectin solution was used overnight to bind the above surface.

- Cell seeding. Cancer cells of epithelial bladder type (T24) were then seeded at a low density. They usually adhered rapidly and spred. The coverglass was attached at the bottom of a $35 \mathrm{~mm}$-culture dish (containing culture medium) in order to carry out microscopic observations. Two types of images were made: a phase contrast one to observe the cell and its contour, and a fluorescent one focused on the beads (at a 
slightly different vertical position). The depth of field of the images was around 1.5 micrometers. Everything was carried out automatically in order to take one set of images at regular time steps ( $2 m n$, for example).

- Microscopy. Images were then collected and treated using the ImageJ software [19], to determine trajectories and/or displacements with respect to the initial position, as explained in the following part. The initial beads position was determined at the end of the experiment by adding distilled water in order to detach the cells allowing a few minutes for the gel to go back to its initial position.

The basic idea in this work is to follow cells as they move in order to obtain displacements, traction stresses, and velocities of migration during a significant duration (usually around one hour or more). This feature is indeed important since most other studies focus on stresses at a given time, but it will be shown here that stresses can vary significantly as cell migration proceeds. Then displacements and tractions will be studied as a function of gel rigidity.

\subsection{Determination of displacements}

The basic step for the calculation of the traction field is the determination of the beads displacements. Essential data for the computation was the cell boundary, the initial beads position and the in-plane displacement of the markers. The displacement field was extracted from a stack of images: the first one showed the beads in the undeformed position and the other images showed the gel deformed under cell traction. Usually a stack of 30 images was used and the times between frames were chosen to be two or three minutes. This enabled to capture sufficient changes in the beads positions but not too much so that beads could be followed along their trajectories.

The processing of these images began with the correction of the images for relative translational shifts. Here we used the ImageJ software and in particular the "Align Slice" plug-in in order to perform a recursive alignment of a stack of images. The alignment proceeded by propagation: each image was used as a template with respect to which the next slice was 
aligned.

From the corrected images sequences, we localized cells and divided images into small areas typically $100 \mu \mathrm{m}$ x $100 \mu \mathrm{m}$, each containing one isolated cell. Beads detection was made using "Particle Tracker", another ImageJ plug-in. The plug-in implemented point detection and a tracking algorithm as described in [32]; it performed two different steps: first the detection of the beads positions in each image and then the beads link into trajectories. The estimation of the bead center location was done by finding the maximum local intensity in the image. The point locations were refined under the assumption that the beads local intensity maxima were near the true geometric centers of the beads and finally spurious detections such as dust or particle aggregates were rejected. The linking algorithm identified centers corresponding to the same physical particle in subsequent frames, using a graph technique theory, and linked these positions into trajectories.

An example of the image processing technique is provided in Fig.9.4; in particular, Figure 9.4(a) represents the fluorescent beads as recorded with the microscope, this configuration being related to the undisturbed position of the markers. Figure 9.4(b) shows the trajectories of these fluorescent markers under cell traction after 70 minutes. They are obtained after treatment of a stack containing 30 images.
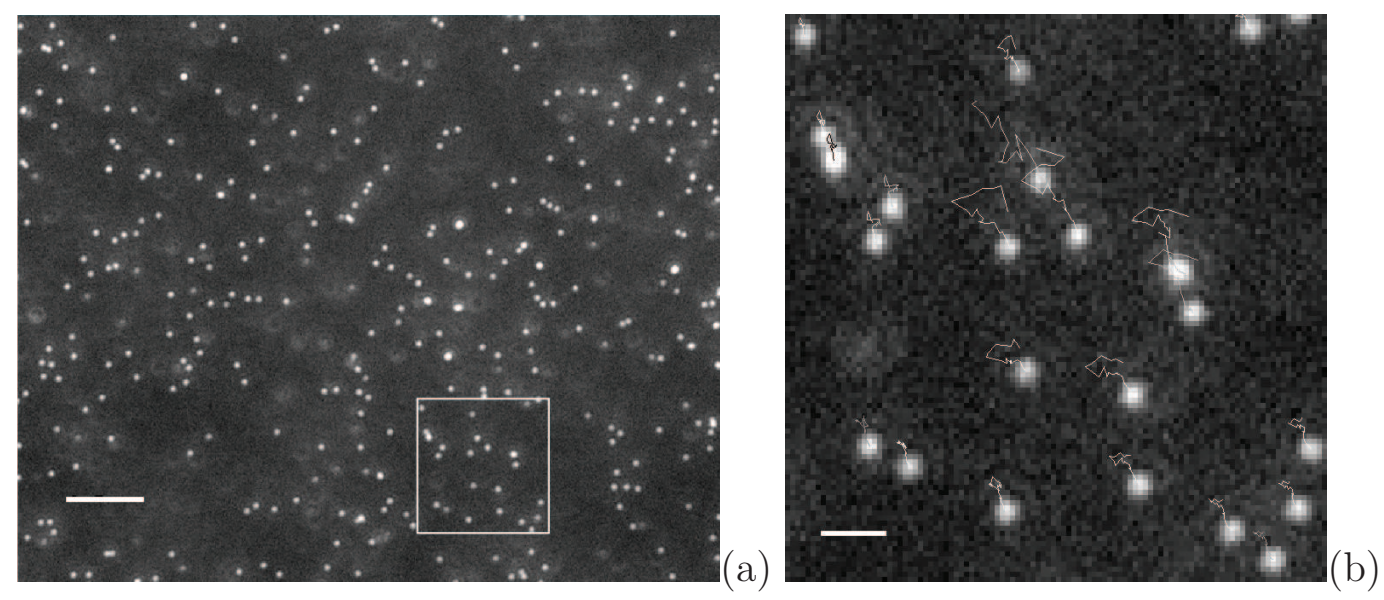

Figure 9.4: (a) Image of undisturbed positions of the fluorescent beads as recorded with the microscope. The scale bar is $10 \mu \mathrm{m}$. (b) Beads trajectories detected with ImageJ after processing a stack containing 30 images. The scale bar is $2 \mu \mathrm{m}$. (b) is a zoom of the square area evidenced in (a). 


\subsection{Determination of traction stresses}

To investigate the traction field generated by T24 cancer cells, we observed cells on different substrates, with a Young's modulus (E) ranging from $2 k P a$ to $10 k P a$. After extracting the beads displacements as described in the previous section, the traction stress field was obtained by solving the system (9.9).
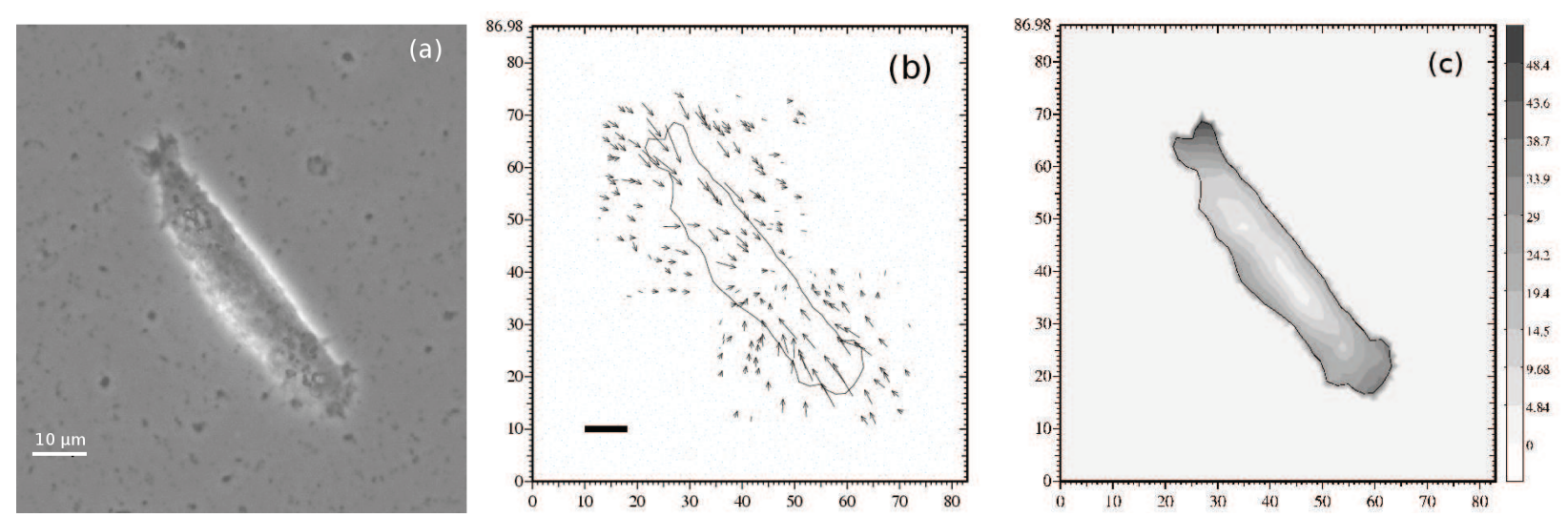

Figure 9.5: (a) T24 cell adhering on a soft polyacrylamide substrate $(E=2 k P a)$. The cell is elongated on the surface. (b) The beads displacements under and around the cell are detected with ImageJ. (c) The traction stresses are shown as a grey-level map for the magnitude. The maximum value is around 50 PicoNewton $/ \mu m^{2}$. The scale bar for displacements is $0.5 \mu \mathrm{m}$ long. The greyscale map is in $\mathrm{pN} / \mu m^{2}$.
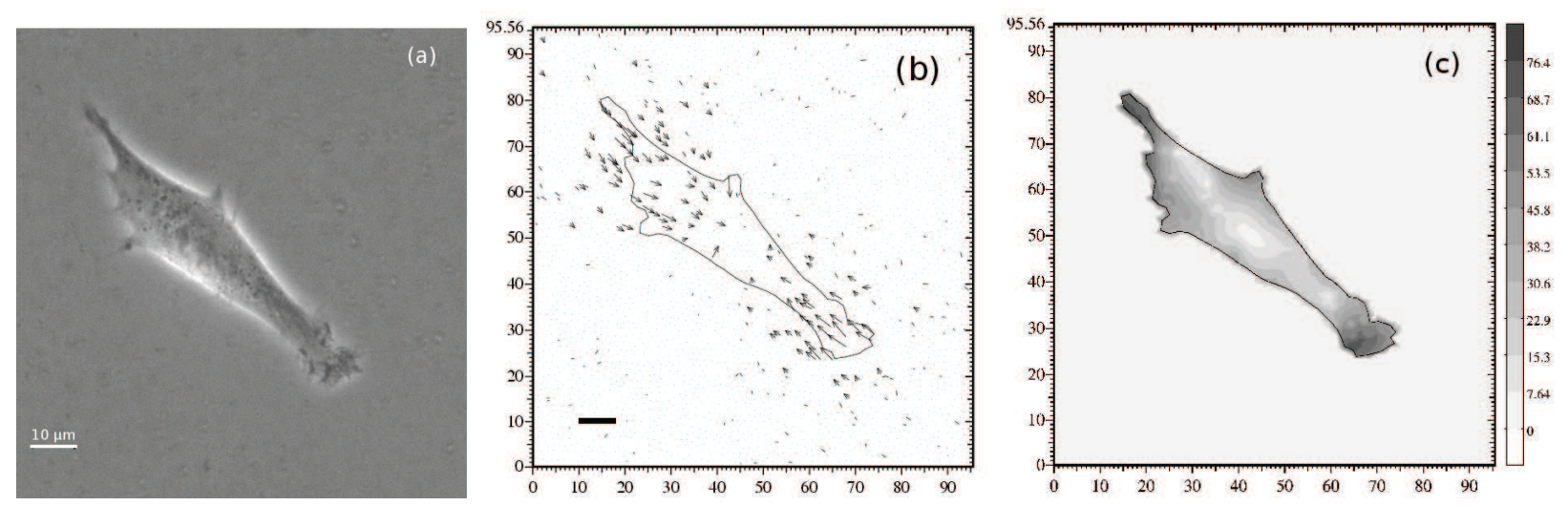

Figure 9.6: (a) T24 cell adhering on a medium stiffness polyacrylamide substrate $(E=$ $6.3 \mathrm{kPa}$ ). The cell looks quite elongated however with small shape protusion compare to Fig.9.5(a). (b) The displacement field has roughly the same magnitude as Fig.9.5. (c) The traction field has a maximum magnitude of about $80 \mathrm{pN} / \mu \mathrm{m}^{2}$. The scale bar for displacements is $0.5 \mu \mathrm{m}$ long. The greyscale map is in PicoNewton $/ \mu m^{2}$.

Fig.9.5(a) shows a T24 cancer cell on the soft gel, $E=2 k P a$. The cell was observed for 

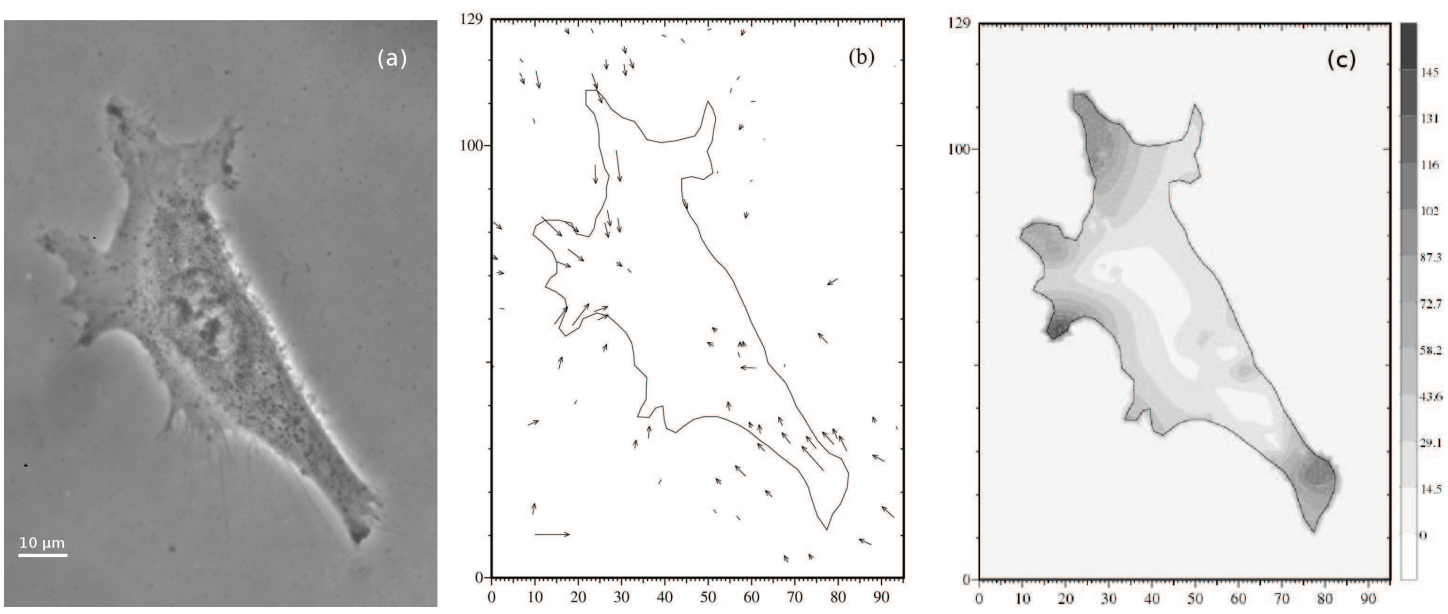

Figure 9.7: (a) T24 cell adhering on a stiff polyacrylamide substrate $(E=10 k P a)$. The cell has a less symmetric shape. (b) The displacement field is of the same magnitude as in Figs 9.5-9.6. (c) The traction field has a maximum magnitude around $145 \mathrm{pN} / \mu^{2}$. The reference vector for displacements is $0.5 \mu \mathrm{m}$ long. The greyscale map is in PicoNewton $/ \mu \mathrm{m}^{2}$.

2 hours and the image was recorded 30 minutes after the beginning of the experiment. The tumor cell is moving on the surface towards the bottom right part of the figure; it is quite elongated on such a surface. There are many beads whose displacements are significant, usually around $0.5 \mu m$; they occur essentially at the cell edges, as seen in Fig.9.5(b). In this same region, the maximum value of the traction stress is obtained, i.e. around $50 \mathrm{pN} / \mu \mathrm{m}^{2}$ as seen in the grey-scale map of figure 9.5(c), representing the traction stress levels.

A T24 cell is shown next on the medium stiff gel, $E=6.3 k P a$ in Fig.9.6(a). After 46 minutes, the cancer cell is quite elongated and exhibits a less symmetric shape than in the previous case. The beads have moved by about $0.5 \mu \mathrm{m}$ maximum (Fig.9.6(b)). The stresses exerted by the tumor cell are shown in Fig.9.6(c): the traction stresses are small along the cell edges with peaks at the front and tail where the maximum magnitude is around $80 \mathrm{pN} / \mu \mathrm{m}^{2}$.

Finally, Fig.9.7(a) shows a T24 cancer cell moving on the stiffer gel, $E=10 k P a$, as well as the related numerical traction field in Fig.9.7(c). The picture has been taken 10 minutes after the beginning of the experiment; at this time the cell exhibits a shape with protrusions and it is anchored by the tail at the bottom right side where the shear stress reaches its maximum value, around $145 \mathrm{pN} / \mu \mathrm{m}^{2}$. In this case, the beads are located close to the cell contour and the beads maximum displacements are still around $0.5 \mu m$, as shown in 
Fig.9.7(b).

From the above, the maximum displacements in these three experiments seem to be in the same range (around $0.5 \mu \mathrm{m}$ ), independently of the rigidity of the substrate.

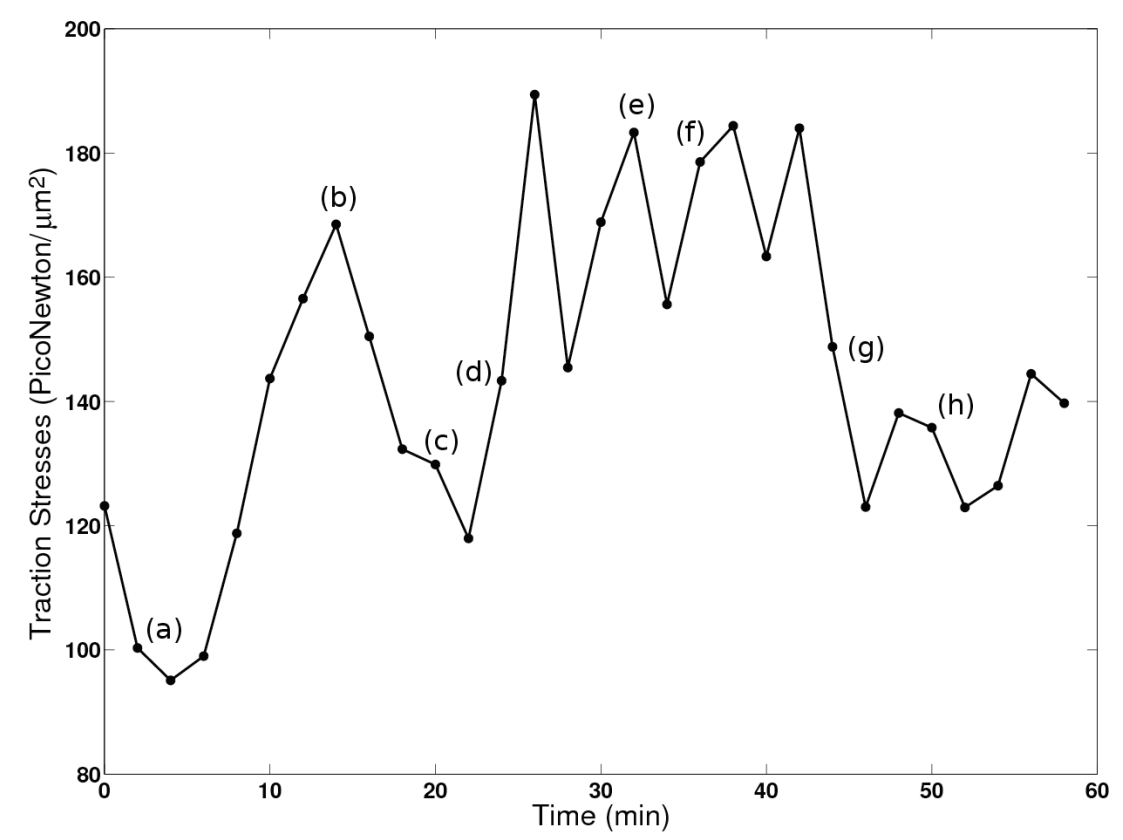

Figure 9.8: Evolution of the maximum value of the traction stresses during T24 cell migration on the rigid substrate $E=10 k P a$.

It is important to study the maximum value of the traction stresses at each time step. For the T24 cancer cell adhering on the rigid substrate, values of the traction stresses were recorded and vary between $90 \mathrm{pN} / \mu \mathrm{m}^{2}$ and $190 \mathrm{pN} / \mu \mathrm{m}^{2}$ as presented in Fig.9.8. Cells do not move in a simple way, but their motion requires the development of protrusions until corresponding stable focal adhesions can be formed. After this is achieved, cells can retract their uropod by pulling on focal adhesions, thus they move in a non continuous way [34]. This is precisely the significance of Fig.9.8 where an unsteady regime of traction stress is observed changing abruptly from one value to another due to rapid pulling on focal adhesion sites. Clearly such mechanisms involved during migration require tight spatial and temporal regulation; we can study this process more accurately in term of traction stresses maps, as proposed in Fig.9.9 on the rigid gel. 

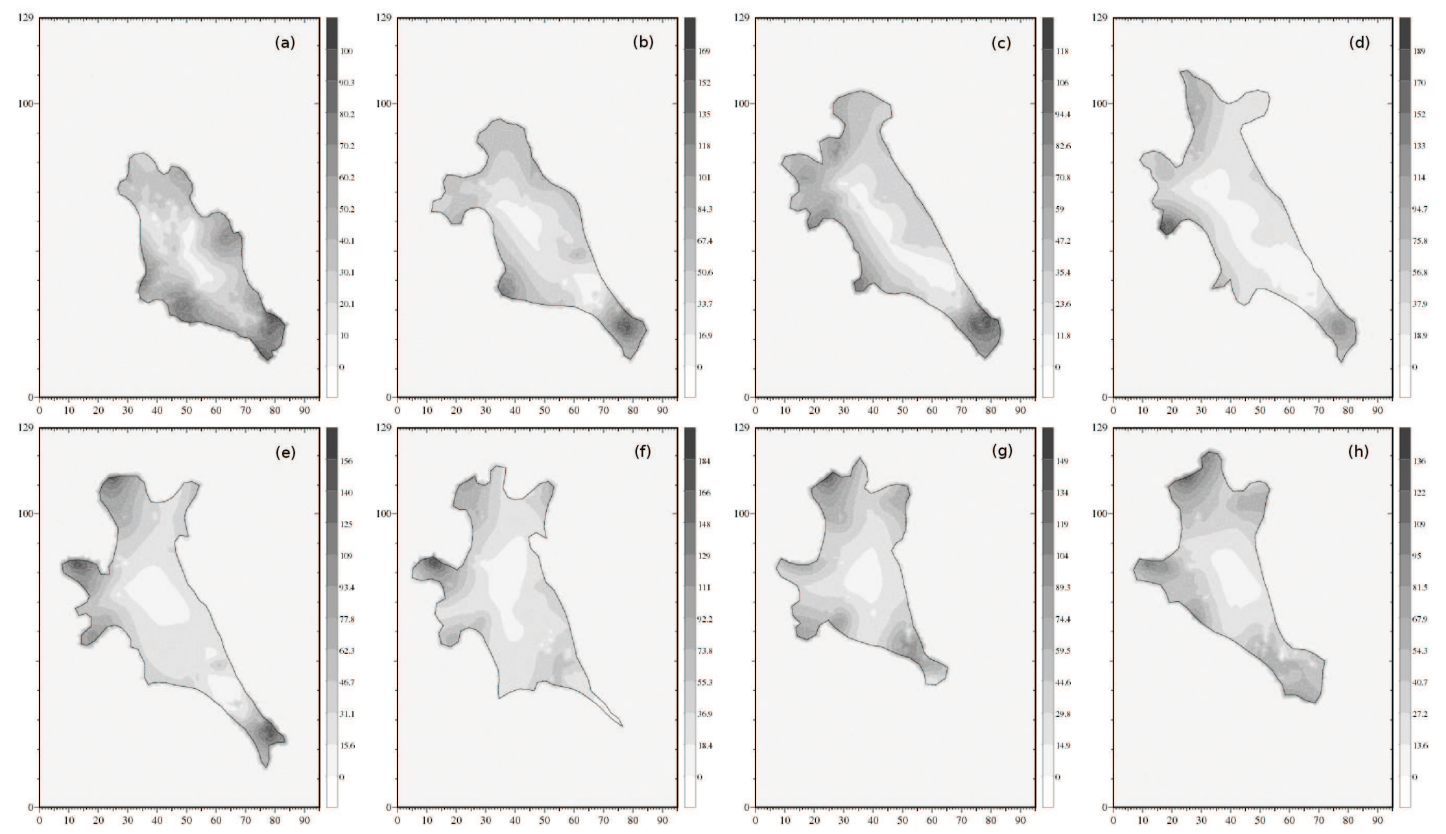

Figure 9.9: Migration of a T24 cell on a stiff gel $(E=10 k P a)$, at time $t=$ 2, 14, 22, 26, 34, 38, 44, $50 \mathrm{mn}$. The cell first adheres strongly (dark region) at its lower right part (a), then starts to exhibit protrusions (b-c-d-e) until it eventually forms new adhesion sites on the upper left of the picture (e-f). At this precise time, it is able to pull on these adhesion sites, contract and detach its rear (f-g); then it starts to move back towards the right direction (h). Note that the greyscale is reset to a range between minimum and maximum values in each frame.

The cell first adheres and binds to the lower right part (a), then it develops protrusions (b-e), as explained above, with the formation of stable adhesion sites (d-f). This corresponds to the dark regions (large stresses). The T24 starts to pull on these new adhesion sites until it moves (f-g). The disassembly of adhesions at the rear of the cell and the retraction of the tail complete the migration picture and enable cell translocation. This mechanism of locomotion for the T24 cancer cell is comparable with the four or five-step picture $[1,34]$ which describes motion of many cell types and is summarized as follows:

- formation of a lamellipodium at the front by actin polymerization;

- development of new focal adhesions coupled with the actin cytoskeleton;

- force traction - cell contraction;

- release of bonds at the rear - Actin and proteins recycling. 
In the next section the observed features are discussed and compared with previous works.

\subsection{Discussion}

The study presented here demonstrates some advantages of the adjoint method [2] when combined with relevant experiments [3]. The major interest is that, instead of solving the CPU-consuming integral equation [10], it directly forces the 2D-averaged problem of partial differential equations to be solved by finite element method. In this respect, the resolution becomes easier, and can be done on a personal computer in a few seconds. The longest part is the data processing of images for the determination of the displacements whatever the method $[7,10,33]$. The results strongly depend on the quality of the images. Further refinements have been made recently as compared to our initial work [3]: pictures are now taken at different heights separated by $0.2 \mu \mathrm{m}$, stacks of about 8-10 pictures are taken at each time step and the best focused images are selected. Thus beads are localized more easily and less beads are lost during the collection of trajectories. Then a good resolution is obtained, as shown by the squared error in Fig.9.1, which is around $10^{-5}$. More precisely, we obtain an error $\left\|\mathbf{u}-\mathbf{u}_{0}\right\|_{2} \sim 3.010^{-3} \mu \mathrm{m}$, which is small compared to displacements in the range $[0.05-0.5 \mu \mathrm{m}]$. This clearly indicates a good resolution as compared to experimental data.

Nevertheless, improvements can still be made: in particular recent works by two groups $[25,30]$ suggest how the resolution can still be enhanced. Merkel et al. [25] use thinner substrates allowing an analytical resolution of the problem. Sabass et al. [30] illustrate the advantage of a combination of complementary fluorescent microscopy in order to capture the location and evolution of focal adhesions, in relation with traction forces, a method also proposed earlier in [4]. This enables to show an increased accuracy of all techniques, in all cases (Boundary Element Method [10], Fourier Transform Traction Cytometry [7], Traction Recovery with Point Forces [33]). In our case, we limit ourselves to the usual traction stress determination but it is possible to combine fluorescence and observe focal adhesions as well as the actin cytoskeleton development. As an example, Fig.9.10 shows the actin cytoskeleton 
organized in stress fibers, in the case of T24 cells adhering on substrates with different rigidities (respectively the ones used before, $E=10-6.3-2 k P a$ ). It is known that stress fibers are colocalized with focal adhesions [1]. In Fig.9.10, most of the stress fibers come close to the cell edges where focal adhesion complexes are usually localized. These photographs clearly indicate that T24 cells form many stress fibers on rigid substrates $(E=10 \mathrm{kPa})$ but less on soft ones $(E=2 k P a)$. The intermediate case also shows stress fibers but they are less numerous as compared to the case of the rigid substrate.
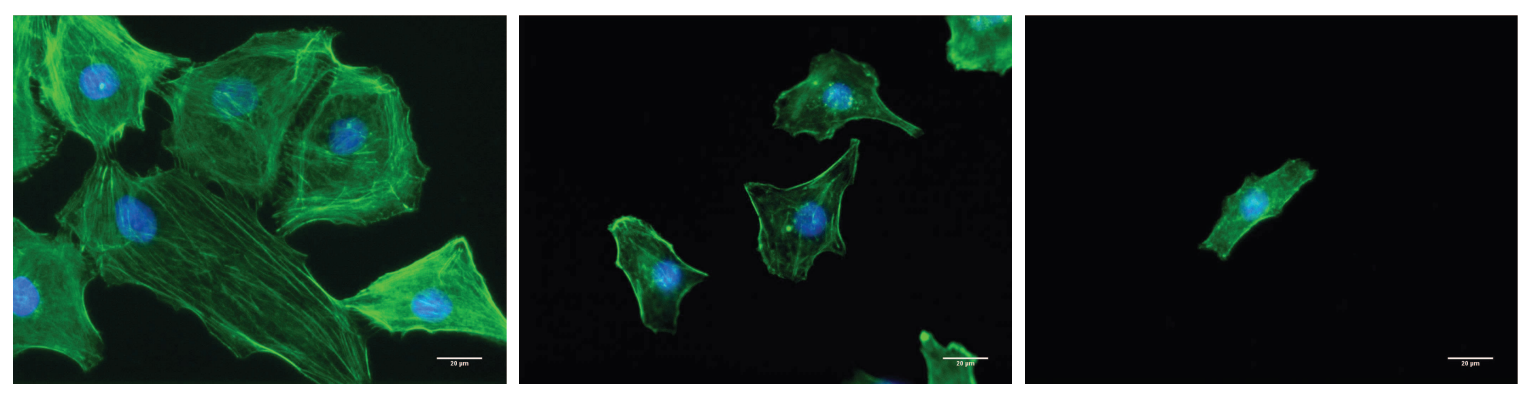

Figure 9.10: T24 cells adhering on substrates with various stiffness $E=10-6.3-2 k P a$ (decreasing from left to right). The actin cytoskeleton has been marked with GFP. Note the formation of stress fibers and a large cell area on the more rigid substrate. In the case of the very soft gel, on the contrary, the actin structure is less developed and the cell barely adheres to the substrate, showing a very small contact area. The scale bar represents $20 \mu \mathrm{m}$.

To continue on the effect of substrate rigidity further, let us concentrate on the stresses exerted by cells on such substrates, a subject already discussed by other authors $[3,11,17,24]$. This current work emphasizes the ability of cells to adapt to a different environment. T24 cancer cells also obey this principle; they develop similar strains when adhering to various rigid substrates. Indeed, the level of deformation does not seem to be affected by substrate rigidity in the relevant range: this comes out from Figs 9.5-9.6-9.7 where it was mentioned that the displacements are very similar (of the order $0.5 \mu \mathrm{m}$ ). Additionally the ratio between maximum stress and Young modulus is almost a constant (strain) of the order 0.02 [3]. This is in agreement with previous works $[11,31]$. It is likely that, as the substrate rigidity increases, such a (stress-rigidity) linear behavior will fail and that there should be a saturation [17] since one expects that cells cannot reinforce their integrin-cytoskeleton links indefinitely [8]. Note that such behaviors can be related to the cell micro-rheological properties [36], in particular their ability to modulate the growth of stress fibers.

It has been also shown that migration of cancer cells on 2D-rigid substrates follows a 
rather classical picture divided into $4-5$ steps. Indeed, Fig.9.8 and Figs 9.9 provide direct evidence of the motion which can be decomposed into these different steps. First, the cancer cell adheres and is not very active in Fig.9.9(a), thus stresses are low. Then it searches for new adhesion sites until it reaches some places that will allow it to establish larger traction stresses. This is precisely where the tractions maxima are obtained in Fig.9.8, at locations (e)-(f). Thanks to these forces, the rear (uropod) can be detached. The cell relaxes again its stresses in Fig.9.9(g) until it will start again to try and move in another direction (Fig.9.9(h)). Therefore, a clear connection has been made between the stress diagram in Fig.9.8 and the migration patterns containing stresses maps in Figs 9.9.

Finally, our study also shows that cell contact area with the substrate increases with substrate rigidity in close correspondance with the development of stress fibers (see Fig.9.10). This fact was only observed previously regarding cell area before [24]. The velocity of migration also varies in the usual way [3]: cells with more stable focal adhesions on rigid substrates definitely move less rapidly as compared to soft substrates, where they move faster because of the difficulties to develop stable adhesions [11].

The order of magnitude of tractions can finally be discussed. T24 cells seem to develop smaller stresses (typically $0.05-0.2 k P a$ here) in the range of the ones found for Human Airway Smooth Muscle (HASM) cells [7]. Such stresses are still much lower than the ones found usually with fibroblasts [10] or endothelial cells [29]. This may be a possible explanation of the reason why cancer cells move rapidly to form metastases and therefore use small traction stresses in order to migrate faster. Still this hypothesis needs to be confirmed in more realistic situations like in vitro three-dimensional migration for example.

\subsection{Conclusions}

The study developed here has revealed some interesting features of cancer cell migration on rigid substrates. It is important to note, that although many studies have already focused on the determination of 
traction forces, the current method is very efficient and shows interesting time dependent features. This method is promising and should allow to investigate new problems related to cancer cells. The ability to distinguish between cell types is of course one first perspective. Secondly, attempts should be made to differentiate invasive from non invasive cancer cells [26]. This could lead to possible diagnosis. Finally, substrate specificity may also be used to compare cell migration characteristics such as their migration velocity or exerted traction stresses. But the most promising follow-up of this work will probably rely in the ability to correlate precisely the cytoskeleton and focal adhesion dynamics with the development of forces exerted by cancer cells. 
246CHAPTER 9. CANCER CELL MIGRATION ON 2D DEFORMABLE SUBSTRATES

\section{References}

[1] B. Alberts et al. (1994) Molecular Biology of the Cell, Third edition, New York, Garland.

[2] D. Ambrosi (2006) Cellular traction as an inverse problem. SIAM J. Appl. Math. 66:2049-2060.

[3] D. Ambrosi, A. Duperray, V. Peschetola, and C. Verdier (2009) Traction patterns of tumor cells. J. Math. Biol. 58:163-181.

[4] N.Q. Balaban, U.S. Schwarz, D. Riveline et al. (2001) Force and focal adhesion assembly: a close relationship studied using elastic micropatterned substrates. Nat. Cell Biol. 3:466-472.

[5] T. Boudou, J. Ohayon, C. Picart and P. Tracqui (2006) An extended relationship for the characterization of Young's modulus and Poisson's ratio of tunable polyacrylamide gels. Biorheology 43:721-728.

[6] K. Burton, J.H. Park and D.L. Taylor (1999) Keratocytes generate traction forces in two phases. Mol. Biol. Cell 10:3745-3769.

[7] J.P. Butler, I.M. Tolic-Norrelykke, B. Fabry and J.J.Fredberg (2002) Traction fields, moments, and strain energy that cells exert on their surroundings. Am. J. Physiol. Cell Physiol. 282:C595-C605.

[8] D. Choquet, D.P. Felsenfeld and M.P. Sheetz (1997) Extracellular matrix rigidity causes strengthening of integrin-cytoskeleton linkages. Cell 88:39-48.

[9] M. Dembo, T. Oliver, A. Ishihara and K. Jacobson (1996) Imaging the traction stresses exerted by locomoting cells with elastic substratum method. Biophys. J. 70:2008-2022.

[10] M. Dembo and Y.L. Wang (1999) Stresses at the cell-to-substrate interface during locomotion of fibroblasts. Biophys. J. 76:2307-2316.

[11] D.E. Discher, P. Janmey and Y. Wang (2005) Tissue cells feel and respond to the stiffness of their substrate. Science 310:1139-1143.

[12] O. du Roure, A. Saez, A. Buguin et al. (2005) Force mapping in epithelial cell migration. Proc. Natl Acad. Sci. USA 102:2390-2395.

[13] A. Engler, L. Bacakova, C. Newman, A. Hategan, M. Griffin and D. Discher (2004) Substrate compliance versus ligand density in cell on gel responses. Biophys. J. 86:617628. 
[14] S. Féréol, R. Fodil, B. Labat et al. (2006) Sensitivity of alveolar macrophages to substrate mechanical and adhesive properties. Cell Motil. Cytoskeleton 63:321-340.

[15] P. Friedl, S. Borgmann and E.B Bröcker (2001) Amoeboid leukocyte crawling through extracellular matrix: lessons from the Dictyostelium paradigm of cell movement. $J$. Leukoc. Biol. 70:491-509.

[16] P. Friedl and K. Wolf (2003) Tumour-cell invasion and migration: diversity and escape mechanisms. Nature 3:362-374.

[17] M. Ghibaudo, A. Saez, L. Trichet et al. (2008) Traction forces and rigidity sensing regulate cell functions. Soft Matter 4:1836-1843.

[18] A.K. Harris, P. Wild and D. Stopak (1980) Silicone rubber substrata: a new wrinkle in the study of cell locomotion. Science 208:177-179.

[19] ImageJ, by Rasband, W.S. 1997-2000. Bethesda, Maryland, USA:U. S. National Institutes of Health. http://rsb.info.nih.gov/ij/.

[20] P.A. Janmey, U. Euteneuer, P. Traub and M. Schliwa (1991) Viscoelastic properties of vimentin compared with other filamentous biopolymer networks. J. Cell Biol. 113: $155-160$.

[21] L. Landau and E. Lisfchitz (1967) Théorie de l'Élasticité , Moscou, Editions Mir.

[22] J. Lee, M. Leonard, T. Oliver, A. Ishihara and K. Jacobson (1994) Traction forces generated by locomoting keratocytes. J. Cell Biol. 127:1957-1964.

[23] J.L. Lions (1968) Contrôle optimal de systèmes gouvernés par des équations aux dérivées partielles, Paris, Dunod et Gauthier-Villard.

[24] C.M. Lo, H.B. Wang, M. Dembo and Y.L. Wang (2000) Cell movement is guided by the rigidity of the substrate. Biophys. J. 79:144-152.

[25] R. Merkel, N. Kirchgessner, C.M. Cesa and B. Hoffmann (2007) Cell force microscopy on elastic layers of finite thickness. Biophys. J. 93:3314-3323.

[26] C.T. Mierke, D.P. Zitterbart, B. Kollmannsberger et al. (2008) Breakdown of the endothelial barrier function in tumor cell transmigration. Biophys. J. 94:2832-2846.

[27] S.P. Palecek, J.C. Loftus, M.H. Ginsberg, D.A.Lauffenburger and A.F. Horwitz (1997) Integrin-ligand binding properties govern cell migration speed through cell-substratum adhesiveness. Nature 385:537-540.

[28] R.J. Pelham and Y. Wang (1997) Cell locomotion and focal adhesions are regulated by 

substrate flexibility. Proc. Natl. Acad. Sci. USA 94:13661-13665.

[29] C.A. Reinhart-King, M. Dembo and D.A. Hammer (2005) The dynamics and mechanics of endothelial cell spreading. Biophys. J. 89:676-689.

[30] B. Sabass, M.L. Gardel, C.M. Waterman and U.S. Schwarz (2008) High resolution traction force microscopy based on experimental and computational advances. Biophys. J. 94:207-220.

[31] A. Saez, A. Buguin, P. Silberzan and B. Ladoux (2005). Is the mechanical activity of epithelial cells controlled by deformations or forces? Biophys. J. 89:L52-L54.

[32] I.F. Sbalzarini and P. Koumoutsakos (2005) Feature point tracking and trajectory analysis for video imaging in cell biology. Struct. Bio. J. 151:182-195.

[33] U.S. Schwarz, N.Q. Balaban, D. Riveline, A. Bershadsky, B. Geiger and S.A. Safran (2002) Calculation of forces at focal adhesions from elastic substrate data: the effect of localized force and the need for regularization. Biophys. J. 83:1380-1394.

[34] M.P. Sheetz, D. Felsenfeld, C.G.Galbraith and D. Choquet (1999) Cell migration as a five-step cycle. Biochem. Soc. Symp. 65:233-243.

[35] J.L. Tan, J. Tien, D.M. Pirone, D.S. Gray, K. Bhadriraju and C.S. Chen (2003) Cells lying on a bed of microneedles: an approach to isolate mechanical force. Proc. Natl Acad. Sci. USA 100:1484-1489.

[36] C. Verdier, Review (2003) Rheological properties of living materials: From cells to tissues. J. Theor. Medicine 5:67-91.

[37] T. Yeung, P.C. Georges, L.A. Flanagan et al. (2005) Effects of substrate stiffness on cell morphology, cytoskeletal structure, and adhesion. Cell Motil Cytoskeleton 60:24-34. 